\title{
Novel Algorithms for Robust Registration of Fiducials in CT and MRI
}

\author{
Sangyoon Lee ${ }^{1}$, Gabor Fichtinger ${ }^{2}$, and Gregory S. Chirikjian ${ }^{1}$ \\ 1 Department of Mechanical Engineering \\ Johns Hopkins University, Baltimore, MD 21218, USA \\ \{sanglee, gregc\}@jhu.edu \\ 2 Center for Computer-Integrated Surgical Systems and Technology \\ Johns Hopkins University, Baltimore, MD 21218, USA \\ gabor@cs.jhu.edu
}

\begin{abstract}
In this paper we present several numerical algorithms for registering fiducials in planar $\mathrm{CT}$ or MRI images to their corresponding three-dimensional locations. The unique strength of these methods is their ability to robustly handle incomplete fiducials patterns, even in extreme cases when as much as one third of the fiducial data is missing from the images. We compare the effectiveness of these algorithms in terms of flops and robustness on actual CT data sets.
\end{abstract}

\section{Introduction}

Our primary objective in this paper is to present robust numerical methods for registration of rigid-body fiducials to the $\mathrm{CT} / \mathrm{MRI}$ image space with the use of a single image slice. A key feature of our methods is that they guarantee reliable registration in situations when conventional methods commonly fail. Our secondary objective is to make these methods applicable to a plurality of conceivable fiducial patterns, without the need for algorithmic refinement or modification.

The primary cause of a failure to register an image slice is a situation when only part of the rigid body fiducial shows up in the resulting image, thus not providing sufficient input data for the registration algorithm. This problem occurs quite frequently during frame-based radiosurgery and neurosurgical planning, when an image slice does not cut across the entire headframe. Thus, only some, but not all, of the fiducial rods are visible in the image. In robotically assisted surgery the end-effector of a robot can be registered to the scanner from a single image slice using a small rigid body fiducial. Most intuitively, a miniature version of a stereotactic head frame is used 8 . Incomplete data tend to be a chronic problem in these systems because it is common for the robot to accidentally move the fiducial frame out of the field of view, causing the image slice to become incomplete for registration. Traditional methods cannot handle this problem without taking extra images, which is not an acceptable solution.

Image guided robots must often work in tight spaces like inside the gantry of a CT or even inside the patient's body, where there is no room for a conventional 
fiducial device composed from a triplet of $\mathrm{V}$-or N-shaped planar motifs. Worse yet, every time when a new fiducial design is introduced, a new registration algorithm and new image processing software has to be developed. Our goal was to devise a method that can cope with an arbitrary pattern of fiducial lines. In this generic scenario, conventional fiducial devices like the BRW [1] or Kelly [2] headframes are uniformly handled, and incomplete scans also fit in the framework. As a byproduct of our robustness analysis, we obtained practical limits for the incompleteness of images (see Section 4).

Stereotactic head frames have been in use for over two decades. Initial applications were intra-cranial neurosurgery and radiosurgery [1, 2, 3]. The methodology has been further extended for extra-cranial radiotherapy applications [4, 5, 6. 7], and then recently for robotically assisted surgery [8]. Popular registration algorithms follow the theme described by Brown 1 and many years later by Susil [8]. Those authors calculate one corresponding point of the image plane from each of the three N-shaped motifs, and reconstruct the image plane from those three calculated points. The main weaknesses of this approach are (1) the inability to handle incomplete patterns when not all fiducial rods leave marks in the image and (2) the inconvenience that the computer software has to be "reinvented" every time the geometry and/or shape of fiducial motifs are modified. Zylka et al. [9] assumed all the image slices have been computed without motion of the head frame between image slices (all slices were parallel), and they registered the lines from the $3 \mathrm{D}$ chunk of image data to the head-frame. They, however, did not solve the problem of registering planar point patterns to lines in space, therefore their approach is not applicable to single sliced based registration.

The mathematical problem in this paper is basically to register a planar image in space, given a set of $n$ known lines in space, given a pattern of $n$ points in a plane, and given a correspondence between the lines and points. The geometrical configuration of lines and image plane is displayed in Figure 1.

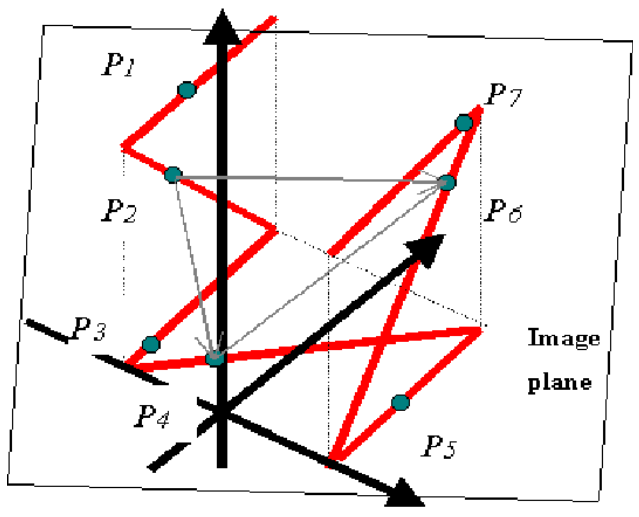

Figure 1: Intersection of image plane.

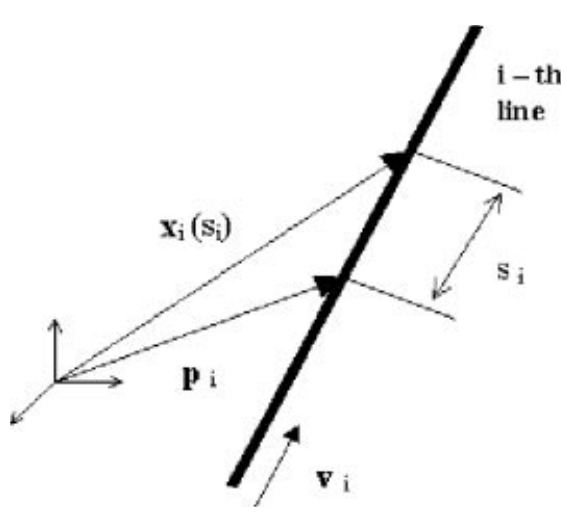

Figure 2: Parameters of a line. 
While the problem of registering one set of points to another has received a lot of attention (see [10] and references therein), the general problem of registering points to lines has not been studied as extensively.

There are two variants on this problem that are addressed here. In the first, we are interested in finding the equation of the plane. If we know this, then the planar registration of one planar set of points to the other can be performed afterwards. In the second formulation of the problem, a frame of reference is attached to the intersecting plane, and we solve for the position and orientation of this frame in three-dimensional space. Hence, in the first approach two threeparameter problems are solved sequentially, while in the second approach one sixparameter problem is solved. We refer to these as the $3 \times 2$ and $6 \times 1$ approaches, respectively. We present several algorithms for each of these approaches.

\section{Algorithms for the $3 \times 2$ Approach}

\subsection{Algorithm 1: Solving a System of Polynomials}

The problem can be solved by simultaneously satisfying $n(n-1) / 2$ constraint equations ( $n$ is the number of lines) of the form in (1)

$$
\left\|\mathbf{x}_{i}\left(s_{i}\right)-\mathbf{x}_{j}\left(s_{j}\right)\right\|^{2}=d_{i j}^{2} .
$$

In general, this problem is overdetermined, so only an approximate solution is possible. As shown in Figure 2, $\mathbf{x}_{i}\left(s_{i}\right)$ is a position vector of a point on the $i$-th line which is defined by the position $\mathbf{p}_{\mathbf{i}}$ and unit direction of $\mathbf{v}_{\mathbf{i}} \cdot s_{i}$ is the arc length from $\mathbf{p}_{\mathbf{i}}$ to $\mathbf{x}_{i}\left(s_{i}\right)$ and $d_{i j}$ is the Euclidean distance between $\mathbf{x}_{i}\left(s_{i}\right)$ and $\mathbf{x}_{j}\left(s_{j}\right)$.

This set of equations will in general be a second-order polynomial in $s_{i}$ and $s_{j}$ of the form:

$$
s_{i}^{2}-2 s_{i} s_{j} \cos \theta_{i j}+s_{j}^{2}+a_{i j} s_{i}+b_{i j} s_{j}=d_{i j}^{2}+c_{i j} .
$$

The constants $a_{i j}, b_{i j}, \theta_{i j}$, and $c_{i j}$ all come from the geometry of the problem and $\cos \theta_{i j}=\mathbf{v}_{\mathbf{i}} \cdot \mathbf{v}_{\mathbf{j}}$.

The approach we take to solve the system of polynomials is to iteratively solve the system by assuming that each of the parameters can vary with "artificial" time. We make an initial guess $s_{i}(0)$ (in practice this guess corresponds to the plane whose normal is the axis of the head frame with value of $c_{i j}$ that causes this plane to cut the head frame in half).

Then we take the time derivative to get

$$
2 s_{i} \dot{s}_{i}-2\left(\dot{s}_{i} s_{j}+s_{i} \dot{s}_{j}\right) \cos \theta_{i j}+2 s_{j} \dot{s}_{j}+a_{i j} \dot{s}_{i}+b_{i j} \dot{s}_{j}=2 d_{i j} \dot{d}_{i j} .
$$

The right-hand-side follows from the fact that $c_{i j}$ is a constant.

We can assemble the $n(n-1) / 2$ equations of the form in (2) as

$$
J(\mathbf{s}) \dot{\mathbf{s}}=\mathbf{w} .
$$


The problem does not dictate the behavior of $\dot{d}_{i j}$ in the mathematical model. However, if we specify

$$
\dot{d}_{i j}=\left(d_{i j}\right)_{\text {measured }}-d_{i j},
$$

where $\left(d_{i j}\right)_{\text {measured }}$ denotes the Euclidean distance between the $i$-th and $j$-th fiducial points on image plane, then iterating (3) with the simple update rule

$$
\mathbf{s}(t+\Delta t)=\mathbf{s}(t)+\Delta t\left(J^{T} J\right)^{-1} J^{T} \mathbf{w}(t),
$$

converges to the solution as long as $\operatorname{det}\left(J^{T} J\right) \neq 0$ for all values of $\mathbf{s}$ encountered during the iterations. Since $s_{i}$ values determine $\mathbf{x}_{i}\left(s_{i}\right)$, we can establish the equation of the plane.

\subsection{Algorithm 2: Look Up Table}

Of course in practice, there is no need to perform the computations described in Algorithm 1 in real time. This algorithm can be used off line to define a look-up table. Given a table of $s_{i}$ values defined on equally spaced values of $d_{i j}$, the corresponding values of $s_{i}$ can be interpolated from the look-up table and the measured values of $d_{i j}$.

\section{Algorithms for the $6 \times 1$ Approach}

\subsection{Algorithm 1: Rate-Linearization of Position and Orientation}

Given the line defined by the position $\mathbf{p}_{\mathbf{i}}$ and unit direction vector $\mathbf{v}_{\mathbf{i}}$, and a point in space $\mathbf{x}_{\mathbf{i}}$, we calculate the Euclidean distance between the line and point by minimizing the cost function

$$
c(s)=\|(\mathbf{p}+s \mathbf{v})-\mathbf{x}\|^{2}=\|\mathbf{p}-\mathbf{x}\|^{2}+2 s \mathbf{v} \cdot(\mathbf{p}-\mathbf{x})+s^{2} .
$$

Setting $d c / d s=0$, we see that the minimizing value of $s$ is $s_{\min }=-\mathbf{v} \cdot(\mathbf{p}-\mathbf{x})$, and so the vector pointing from $\mathbf{x}$ to the closest point on the line is

$$
\mathbf{h}=(\mathbf{p}-\mathbf{x})-[\mathbf{v} \cdot(\mathbf{p}-\mathbf{x})] \mathbf{v},
$$

which we can write as $\mathbf{h}=(\mathbf{p}-\mathbf{x})-\mathbf{v v}^{T}(\mathbf{p}-\mathbf{x})=\left[\mathbb{I}-\mathbf{v} \mathbf{v}^{\mathrm{T}}\right](\mathbf{p}-\mathbf{x})$.

We can formulate the problem as finding the rigid-body trajectory $(R(t), \mathbf{b}(t))$ such that $\mathbf{x}_{i}(t)=R(t) \mathbf{y}_{i}+\mathbf{b}(t)$ drives each of the vectors

$$
\left[\mathbb{I}-\mathbf{v}_{\mathrm{i}} \mathbf{v}_{\mathrm{i}}^{\mathrm{T}}\right]\left(\mathbf{p}_{\mathrm{i}}-\mathbf{x}_{\mathrm{i}}(\mathrm{t})\right)=\boldsymbol{\delta}_{\mathbf{l}}(\mathrm{t})
$$

to zero, where $\mathbf{y}_{i}$ denotes the coordinates of $i$-th fiducial in the image plane. If this can be accomplished, it means that each of the fiducials is driven to its corresponding element of the three-dimensional cage.

Note that the matrix $\left[\mathbb{I}-\mathbf{v v}^{\mathrm{T}}\right]$ is not invertible since $\|\mathbf{v}\|=1$ implies that the matrix $\mathbf{v v}^{T}$ has an eigenvalue equal to one corresponding to eigenvector $\mathbf{v}$. 
Making the substitution $\mathbf{x}_{i}(t)=R(t) \mathbf{y}_{i}+\mathbf{b}(t)$ in (4) and rearranging terms we see that

$$
\left[\mathbb{I}-\mathbf{v}_{\mathrm{i}} \mathbf{v}_{\mathrm{i}}^{\mathrm{T}}\right] \mathbf{p}_{\mathrm{i}}-\left[\mathbb{I}-\mathbf{v}_{\mathrm{i}} \mathbf{v}_{\mathrm{i}}^{\mathrm{T}}\right]\left(\mathrm{R} \mathbf{y}_{\mathrm{i}}+\mathbf{b}\right)=\boldsymbol{\delta}_{\mathbf{l}}(\mathrm{t}) .
$$

We now take the derivative of both sides with respect to artificial time, and observe that $\mathbf{p}_{i}$ and $\mathbf{v}_{i}$ are constant vectors. If in addition we observe that

$$
\dot{R} \mathbf{y}_{i}=\dot{R} R^{T} R \mathbf{y}_{i}=\boldsymbol{\omega} \times\left(R \mathbf{y}_{i}\right)=-\left(R \mathbf{y}_{i}\right) \times \boldsymbol{\omega}
$$

where $\boldsymbol{\omega}=\operatorname{vect}\left(\dot{R} R^{T}\right)$, then we can write

$$
\left[\mathbb{I}-\mathbf{v}_{\mathrm{i}} \mathbf{v}_{\mathrm{i}}^{\mathrm{T}}\right]\left[\operatorname{matr}\left(\mathrm{R} \mathbf{y}_{\mathrm{i}}\right),-\mathbb{I}\right]\left[\boldsymbol{\omega}^{\mathrm{T}}, \dot{\mathbf{b}}^{\mathrm{T}}\right]^{\mathrm{T}}=\dot{\boldsymbol{\delta}} .
$$

Here matr ( ) denotes that for a vector $\mathbf{z}, \operatorname{matr}(\mathbf{z})$ is the skew symmetric matrix such that $\operatorname{matr}(\mathbf{z}) \mathbf{x}=(\mathbf{z}) \times \mathbf{x}$. In other words, $\operatorname{vect}(\operatorname{matr}(\mathbf{z}))=(\mathbf{z})$.

If we force $\dot{\boldsymbol{\delta}}_{i}$ to zero by defining

$$
\dot{\delta}_{i}=-\alpha \boldsymbol{\delta}_{i}
$$

for some positive constant $\alpha$, then $\boldsymbol{\omega}$ and $\dot{\mathbf{b}}$ can be solved for at each value of time along the way by inverting the over constrained system resulting from concatenating (5) for $i=1,2, \ldots, n$. Once this is done, the values of $\mathbf{b}(t)$ and $R(t)$ can be updated using the rules

$$
\mathbf{b}(t+\Delta t)=\mathbf{b}(t)+\Delta t \dot{\mathbf{b}}(t)
$$

and

$$
R(t+\Delta t)=[\mathbb{I}+\Delta \mathrm{t} \operatorname{matr}(\boldsymbol{\omega})(\mathrm{t}))] \mathrm{R}(\mathrm{t}) .
$$

Since the rotational updates have the potential to cause $R(t)$ to stray from being a rotation matrix, the occasional renormalization

$$
R(t) \rightarrow R(t)\left(R^{T}(t) R(t)\right)^{-1}
$$

may be required. This step could be replaced by finding the Euler angles or Cayley parameters that best approximate $R(t)$, then replace $R(t)$ with the resulting rotation matrix.

We note that if the actual rotation matrix is close to being the identity matrix (as will be the case when the image plane is close to cutting the cage straight on), then only one iteration may be required.

\subsection{Algorithm 2: Minimization over Position, Orientation, and Arc Lengths}

Given the coordinates $\left\{\mathbf{y}_{i}\right\}$ of fiducials in the image plane, we can simultaneously solve for the position and orientation of a reference frame attached to the image plane and the arc lengths $\left\{s_{i}\right\}$ as follows.

We observe that

$$
\mathbf{p}_{i}+s_{i} \mathbf{v}_{i}=R \mathbf{y}_{i}+\mathbf{b} .
$$


Let us assume that we have an initial guess of the orientation of the frame attached to the image plane, and that the actual orientation is not very different than this initial guess. Then we write

$$
R=R_{0}(\mathbb{I}+\Omega) .
$$

Here $\Omega=-\Omega^{T}$ has entries that are small. The vector $\boldsymbol{\omega}$ is defined such that

$$
\boldsymbol{\omega} \times \mathbf{x}=\Omega \mathbf{x}
$$

for any $\mathbf{x} \in \mathbb{R}^{3}$, This means we can rewrite (6) as

$$
\mathbf{p}_{i}=-s_{i} \mathbf{v}_{i}+R_{0} \mathbf{y}_{i}-R_{0}\left(\mathbf{y}_{i} \times \boldsymbol{\omega}\right)+\mathbf{b} .
$$

By defining $Y_{i}$ to be the matrix such that $Y_{i} \mathbf{x}=\mathbf{y}_{i} \times \mathbf{x}$ for every $\mathbf{x} \in \mathbb{R}^{3}$, it follows that we can write the following linear equation:

$$
\mathbf{p}_{i}-R_{0} \mathbf{y}_{i}=\left[\mathbf{0}, \ldots, \mathbf{0},-\mathbf{v}_{i}, \mathbf{0}, \ldots, \mathbf{0},-R_{0} Y_{i}, \mathbb{I}\right]\left[\begin{array}{l}
\mathbf{s} \\
\boldsymbol{\omega} \\
\mathbf{b}
\end{array}\right]
$$

where

$$
\mathbf{s}=\left[0, \ldots, 0, s_{i}, 0, \ldots, 0\right]^{T} .
$$

Stacking these equations on top of each other for $i=1, \ldots, n$ results in a system of $(3 n) \times(n+6)$ scalar equations in $n+6$ parameters $(n$ arc lengths and 6 rigidbody motion parameters). This can be solved in the least-squares sense using a pseudo-inverse.

\section{Numerical Results and Experimental Data}

The algorithms that are described in the previous sections are applied to real CT data. Among commonly used stereotactic devices, the BRW and Kelly frames are used. The comparison of the algorithms is made in terms of the number of flops and the robustness. We use 44 and 9 image slices which are acquired from the BRW frame and the Kelly frame, respectively.

It is observed that the application of algorithm 1 for the $3 \times 2$ approach generates convergent $s_{i}$ values. Figure 3 demonstrates the convergence of $s_{i}$ values for an image slice from the Kelly frame. It is also observed that the error converges to zero as the number of iterations increases in algorithm 1 for the $3 \times 2$ approach and algorithms 1 and 2 for the $6 \times 1$ approach. Figure 4 displays the convergence of error to zero for an image slice from the BRW frame in the application of the algorithm 1 for the $6 \times 1$ approach.

The numbers in Table 1 are the average value of the results for all the tested image slices. It is observed that algorithm 1 for the $3 \times 2$ approach is superior to the other algorithms in terms of flops. Table 1 also demonstrates the comparison of algorithms 1 and 2 for the $3 \times 2$ approach. Three-dimensional linear interpolation is used in the look-up table algorithm. Interestingly, $1 \mathrm{~mm}$ and $0.1 \mathrm{~mm}$ 

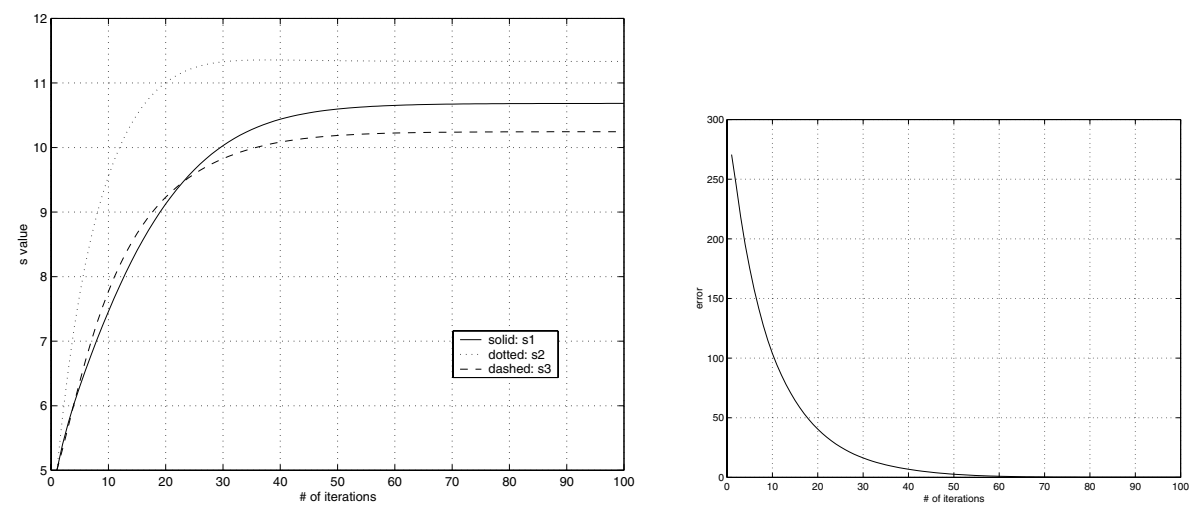

Figure 3: $s_{i}$ values vs. No. of iterations. Figure 4: error vs No. of iterations.

Table 1. Comparison of algorithms in terms of flops end error

\begin{tabular}{|c|c|c|}
\hline \multirow{2}{*}{ algorithms } & \multirow{2}{*}{ flops } & comparison to 9 rod case $(100 \%)$ \\
\hline & & \begin{tabular}{|l|}
8 rods 7 rods 6 rods 5 rods \\
\end{tabular} \\
\hline algorithm 1 for $3 \times 2$ & 24126 & 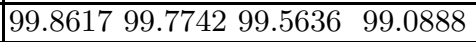 \\
\hline algorithm 2 for $3 \times 2$ & 61504 & \\
\hline algorithm 1 for $6 \times 1$ & 332113 & $99.899799 .896399 .3482 \quad 98.4203$ \\
\hline algorithm 2 for $6 \times 1$ & 455384 & $99.864099 .852999 .6326 \quad 98.6278$ \\
\hline
\end{tabular}

increments in $d_{i j}$ result nearly the same accuracy. Due to limited disk space and time for the generation of the table, a $50 \times 50 \times 50$ table is used. The look-up table algorithm does not show advantage in flops despite a small size of table, thus we omitted this method from further testing.

We tested the robustness of three algorithms to missing fiducial marks. Theoretically, as few as 3 slanted rods for the Kelly frame and 4 rods for the BRW frame are necessary to reach convergence. However, in real surgical cases we have never observed less than 6 marks when using such frames. All those three algorithms are found stable with 8,7,6 and even 5 markers. Table 1 shows that less than $1.6 \%$ difference in parameter values is found between cases where all 9 markers are used and only 5 markers are used. The best performing method in this aspect too was algorithm 1 for the $3 \times 2$ approach.

\section{Summary and Conclusions}

In this paper we have presented a number of different techniques for determining the spatial location of fiducial features in planar images. The two broad categories of algorithms that we developed are classified according to (1) whether they first seek the parameters describing the image plane, and then register within that plane or (2) whether they register the planar points to the three di- 
mensional fiducials directly. The strengths of our methods are (1) the ability to handle incomplete fiducial patterns and (2) the applicability to a plurality of conceivable fiducial patterns without algorithmic modification. The algorithms are sufficiently robust to handle as few as 6 fiducials (out of 9) and are applicable in all current robotically assisted percutaneous applications (prostate, kidney, liver, spine) under development at the Johns Hopkins University. These algorithms are expected to be useful in clinical applications where robot end-effectors or conventional surgical devices need to be registered to CT or MRI images. Ongoing research focuses on two additional aspects: (1) statistical analysis of robustness of the algorithms to noise possibly occurring in the acquisition of image; and (2) analysis of adaptability of the algorithms to headframes composed of curves, e.g. helices.

\section{References}

[1] Brown R. A., Roberts T. S., and Osborne A. G., "Stereotactic frame and computer software for CT-directed neurosurgical localization," Invest. Radiol., Vol. 15, pp. 308-312, 1980.

[2] Goers S., et al, "A computed tomographic stereotactic adaptation system," Neurosurgery, Vol. 10, pp. 375-379, 1982.

[3] Leksell L. and Jerenberg B., "Stereotaxis and tomography: a technical note," Acta Neurochir, Vol. 52, pp. 1-7, 1980.

[4] Lax I, et al. "Stereotactic radiotherapy of malignancies in the abdomen. Methodological aspects.," Acta Oncol., Vol. 33, No. 6, pp.677-83, 1994.

[5] Takacs I and Hamilton AJ, "Extracranial stereotactic radiosurgery: applications for the spine and beyond," Neurosurg Clin N Am, Vol. 10, No. 2, pp.257-70, April 1999.

[6] Lohr F, et al., "Noninvasive patient fixation for extracranial stereotactic radiotherapy," Int J Radiat Oncol Biol Phys, Vol. 45, No. 2, pp. 521-7, September 1999.

[7] Erdi Y., Wessels B., DeJager R., Erdi A., et al., "A new fiducial alignment system to overlay abdominal $\mathrm{CT}$ or MR images with radiolabeled antibody SPECT scans," Cancer, 73 (3 Suppl), pp. 923-931, 1994.

[8] R. Susil, J. Anderson, R. Taylor, "A single image registration method for CT guided interventions," Proceedings to MICCAI'99, Lecture notes in Computer Science, Vol. 1679, pp 798-808, Springer 1999.

[9] Zylka W, Sabczynski J, Schmitz G, "A Gaussian approach for the calculation of the accuracy of stereotactic frame systems," Medical Physics, Vol. 26, No. 3, pp. 381-391, March 1999.

[10] Chirikjian, G.S., Kyatkin, A.B., Engineering Applications of Noncommutative Harmonic Analysis, CRC Press, October 2000. 